\title{
ON CERTAIN LIMIT THEOREMS OF THE THEORY OF PROBABILITY
}

P. ERDÖS AND M. KAC

1. Introduction. In this paper we prove the following four limit theorems:

Let $X_{1}, X_{2}, X_{3}, \cdots$ be independent identically distributed random ${ }^{1}$ variables each having mean 0 and standard deviation 1. Let

$$
s_{k}=X_{1}+X_{2}+\cdots+X_{k}
$$

then:

I.

$$
\lim _{n \rightarrow \infty} \text { prob. }\left\{\max \left(s_{1}, s_{2}, \cdots, s_{n}\right)<\alpha n^{1 / 2}\right\}=\sigma_{1}(\alpha)
$$

where

$$
\sigma_{1}(\alpha)=0 \quad(\alpha \leqq 0)
$$

and

$$
\sigma_{1}(\alpha)=\left(\frac{2}{\pi}\right)^{1 / 2} \int_{0}^{\alpha} \exp \left(-\frac{u^{2}}{2}\right) d u \quad(\alpha \geqq 0) .
$$

II.

$$
\lim _{n \rightarrow \infty} \text { prob. }\left\{\max \left(\left|s_{1}\right|,\left|s_{2}\right|, \cdots,\left|s_{n}\right|\right)<\alpha n^{1 / 2}\right\}=\sigma_{2}(\alpha)
$$

where

$$
\sigma_{2}(\alpha)=\frac{4}{\pi} \sum_{m=0}^{\infty} \frac{(-1)^{m}}{2 m+1} \exp \left\{-(2 m+1)^{2} \pi^{2} / 8 \alpha^{2}\right\} \quad(\alpha \geqq 0)
$$

III.

$$
\lim _{n \rightarrow \infty} \text { prob. }\left\{\frac{s_{1}^{2}+s_{2}^{2}+\cdots+s_{n}^{2}}{n^{2}}<\alpha\right\}=\sigma_{3}(\alpha)
$$

where

Received by the editors September 12, 1945, and, in revised form, December 3, 1945.

${ }^{1}$ This condition can be replaced by a weaker one. In fact, it is enough to assume that the $X$ 's are such that the central limit theorem is applicable. 
$\sigma_{3}(\alpha)=\frac{\pi^{-3 / 2}}{4} \int_{0}^{\alpha / 2} d u u^{-8 / 2} \int_{0}^{\pi / 2}(\cos t)^{-1 / 2} \theta_{1}^{\prime}\left(\frac{t}{2}, e^{-1 / 4 u}\right) d t \quad(\alpha \geqq 0)$

and

$$
\theta_{1}(z, q)=2 \sum_{n=0}^{\infty}(-1)^{n} q^{(2 n+1)^{2} / 4} \sin (2 n+1) z, \quad \theta_{1}^{\prime}=\frac{\partial}{\partial z} \theta_{1} .
$$

IV.

$$
\lim _{n \rightarrow \infty} \text { prob. }\left\{\frac{\left|s_{1}\right|+\left|s_{2}\right|+\cdots+\left|s_{n}\right|}{n^{3 / 2}}<\alpha\right\}=\sigma_{4}(\alpha),
$$

where the real Laplace transform of $\sigma_{1}(\alpha)$ is given by the formula

$$
\int_{0}^{\infty} e^{-z \alpha} d \sigma_{4}(\alpha)=\sum_{j=1}^{\infty} \kappa_{j} \exp \left(-\delta_{j} z^{2 / 8}\right) \quad(z>0),
$$

and where $\delta_{j}$ is the $j$ th positive root of the derivative of

$$
P(y)=\frac{(2 y)^{1 / 2}}{3}\left\{J_{1 / 3}\left(\frac{2^{3 / 2}}{3} y^{3 / 2}\right)+J_{-1 / 3}\left(\frac{2^{3 / 2}}{3} y^{3 / 2}\right)\right\}
$$

and

$$
\kappa_{j}=\frac{1+3 \int_{0}^{\delta j} P(y) d y}{3 \delta_{j} P\left(\delta_{j}\right)}
$$

The proofs of all these theorems follow the same pattern. It is first proved that the limiting distribution exists and is independent of the distribution of the $X$ 's; then the distribution of the $X$ 's is chosen conveniently so that the limiting distribution can be calculated explicitly.

This simple principle has, to the best of our knowledge, never been used before except in a paper by one of the authors in which IV is proved in all detail. ${ }^{2}$

Theorems I and II generalize and simplify several results of $\mathrm{Ba}$ chelier. ${ }^{3}$ Bachelier's work in spite of being both inspired and impor-

$2 \mathrm{M}$. Kac, On the average of a certain Wiener functional and a related limit theorem in calculus of probability, to appear in Trans. Amer. Math. Soc. vol. 59 (1946).

${ }^{3}$ L. Bachelier, Les lois des grands nombres du calcul de probabilités, Paris, GauthierVillars, 1937. See in particular $\$ \$ 18,21,22,35$. This book contains no proofs but it gives references to earlier papers. For the modern and rigorous approach to these questions, based on the differential equation of diffusion, see the third and fourth chapters of A. Khintchine's book Asymptotische Gesetze der Wahrscheinlichkeitsrecinung, Berlin, Springer, 1933. Our proofs, however, are entirely elementary and do not depend on the use of parabolic differential equations. 
tant does not always satisfy the modern requirements of rigor. His methods often depend on replacing difference equations by differential equations, a step not always easy to justify.

The limiting distribution of III was discovered by Cameron and Martin in their work on Wiener space. ${ }^{4}$ Their result is equivalent to the conclusion of III if the X's are assumed to be normally distributed. We use here a different method and make our considerations independent of the use of Wiener space. The Laplace transform of $\sigma_{4}(\alpha)$ has been recently calculated by Kac. ${ }^{5}$ There seems to be very little hope that a reasonably simple expression for $\sigma_{4}(\alpha)$ itself can be found.

2. Proofs of I and II. Let $G_{1}, G_{2}, G_{3}, \cdots$ be independent, normally distributed random variables each having mean and standard deviation 1 and let

$$
R_{k}=G_{1}+G_{2}+\cdots+G_{k} .
$$

Let furthermore $\epsilon>0$ and

$$
P_{n}(\alpha)=\text { prob. }\left\{\max \left(s_{1}, s_{2}, \cdots, s_{n}\right)<\alpha n^{1 / 2}\right\} .
$$

We first prove that for every integer $k$ we have

$$
\text { prob. } \begin{aligned}
\left\{\max \left(R_{1}, \cdots, R_{k}\right)\right. & \left.<(\alpha-\epsilon) k^{1 / 2}\right\}-\frac{1}{\epsilon^{2} k} \leqq \liminf _{n \rightarrow \infty} P_{n}(\alpha) \\
\leqq & \limsup _{n \rightarrow \infty} P_{n}(\alpha) \leqq \text { prob. }\left\{\max \left(R_{1}, \cdots, R_{k}\right)<\alpha k^{1 / 2}\right\} .
\end{aligned}
$$

Let

and

$$
n_{j}=\left[j \frac{n}{k}\right] \quad(j=0,1,2, \cdots, k),
$$

$$
P_{n, k}(\alpha)=\text { prob. }\left\{\max \left(s_{n_{1}}, s_{n_{2}}, \cdots, s_{n_{k}}\right)<\alpha n^{1 / 2}\right\} .
$$

It follows immediately from the multidimensional central limit theorem that

$$
\lim _{n \rightarrow \infty} P_{n, k}(\alpha)=\text { prob. }\left\{\max \left(R_{1}, \cdots, R_{k}\right)<\alpha k^{1 / 2}\right\} .
$$

${ }^{4}$ R. H. Cameron and W. T. Martin, The Wiener measure of Hilbert neighborhoods in the space of real continuous functions, Journal of Mathematics and Physics vol. 23 (1944) pp. 195-209.

${ }^{5}$ Loc. cit. footnote 2 . It should be mentioned that the actual computation of the Laplace transform of $\sigma_{4}(\alpha)$ forms the major part of the proof of IV. 
Let

$$
E_{r}=\text { prob. }\left\{s_{r} \geqq \alpha n^{1 / 2}, s_{1}<\alpha n^{1 / 2}, \cdots, s_{r-1}<\alpha n^{1 / 2}\right\} .
$$

We see immediately that

$$
\sum_{r=1}^{n} E_{r}=1-P_{n}(\alpha) \leqq 1
$$

For $n_{i}<r \leqq n_{i+1}$ we write

$E_{r}=$ prob. $\left\{s_{r} \geqq \alpha n^{1 / 2}, s_{1}<\alpha n^{1 / 2}, \cdots, s_{r-1}<\alpha n^{1 / 2},\left|s_{n_{i+1}}-s_{r}\right| \geqq \epsilon n^{1 / 2}\right\}$

+prob. $\left\{s_{r} \geqq \alpha n^{1 / 2}, s_{1}<\alpha n^{1 / 2}, \cdots, s_{r-1}<\alpha n^{1 / 2},\left|s_{n_{i+1}}-s_{r}\right|<\epsilon n^{1 / 2}\right\}$.

The first of these probabilities is obviously equal to $E_{r}$ prob. $\left\{\mid s_{n_{i+1}}\right.$ $\left.-s_{r} \mid \geqq \epsilon n^{1 / 2}\right\}$ and hence by Tchebychef's inequality it is less than

$$
E_{r} / k \epsilon^{2} \text {. }
$$

Thus

$$
\begin{aligned}
1-P_{n}(\alpha) \leqq \frac{1}{k \epsilon^{2}}+\sum_{i} \sum_{n_{i}<r \leqq n_{i+1}} \text { prob. }\left\{s_{r} \geqq \alpha n^{1 / 2}, s_{1}<\alpha n^{1 / 2},\right. \\
\left.\cdots, s_{r-1}<\alpha n^{1 / 2},\left|s_{n_{i+1}}-s_{r}\right|<\epsilon n^{1 / 2}\right\} .
\end{aligned}
$$

The double sum is obviously less than the probability that at least one of the sums $s_{n_{1}}, s_{n_{2}}, \cdots, s_{n_{k}}$ is greater than $(\alpha-\epsilon) n^{1 / 2}$. Hence

$$
1-P_{n}(\alpha)<\frac{1}{k \epsilon^{2}}+1-P_{n, k}(\alpha-\epsilon),
$$

and since $P_{n}(\alpha)<P_{n, k}(\alpha)$ we obtain.

$$
P_{n, k}(\alpha-\epsilon)-1 / k \epsilon^{2}<P_{n}(\alpha)<P_{n, k}(\alpha) \text {. }
$$

Letting $n \rightarrow \infty$ and using (2) we obtain (1). Let us now consider the particular case in which

$$
\text { prob. }\left\{X_{j}=1\right\}=\text { prob. }\left\{X_{j}=-1\right\}=1 / 2 \text {. }
$$

For these random variables the problem becomes the classical problem of the "ruin of the player" and it is well known" that I holds. Thus applying (1) to this particular case we get

$$
\begin{array}{r}
\text { prob. }\left\{\max \left(R_{1}, \cdots, R_{k}\right)<(\alpha-\epsilon) k^{1 / 2}\right\}-1 / \epsilon^{2} k \\
\leqq \sigma_{1}(\alpha) \leqq \text { prob. }\left\{\max \left(R_{1}, \cdots, R_{k}\right)<\alpha k^{1 / 2}\right\}
\end{array}
$$

' See for instance R. v. Mises, Wahrscheinlichkeitsrechnung, Leipzig and Vienna, F. Deuticke, 1931. In particular pp. 499-506. 
Replacing $\alpha$ by $\alpha+\epsilon$ we obtain

$$
\text { prob. }\left\{\max \left(R_{1}, \cdots, R_{k}\right)<\alpha k^{1 / 2}\right\} \leqq \sigma_{1}(\alpha+\epsilon)+1 / \epsilon^{2} k
$$

and hence, using (1), for the general case

$\sigma_{1}(\alpha-\epsilon)-1 / \epsilon^{2} k \leqq \liminf _{n \rightarrow \infty} P_{n}(\alpha) \leqq \limsup _{n \rightarrow \infty} P_{n}(\alpha) \leqq \sigma_{1}(\alpha+\epsilon)+1 / \epsilon^{2} k$.

We complete the proof of I by letting $k \rightarrow \infty$ (while keeping $\epsilon$ fixed) and by noting that $\sigma_{1}(\alpha)$ is continuous.

The proof of II proceeds in exactly the same manner. The only difference comes in calculating

$$
\lim _{n \rightarrow \infty} \text { prob. }\left\{\max \left(\left|s_{1}\right|, \cdots,\left|s_{n}\right|\right)<\alpha n^{1 / 2}\right\}
$$

for the special random variables (3). The fact that in this case one is led to $\sigma_{2}(\alpha)$ is again implied by the classical theory. ${ }^{7}$

We should like to call the reader's attention to the fact that (4) provides a convenient and strong estimate for $P_{k}(\alpha)$ in the case where the $X$ 's are normally distributed. A similar estimate can be obtained by writing out in detail the proof of II.

3. Proofs of III and IV. Let $n_{1}, n_{2}, \cdots, n_{k}$ have the same meaning as in $\$ 2$. Consider the difference

$$
D_{n}=\frac{1}{n^{2}} \sum_{r=1}^{n} s_{r}^{2}-\frac{1}{n^{2}} \sum_{i=1}^{k}\left(n_{i}-n_{i-1}\right) s_{n_{i}}^{2}
$$

We have

$$
\left|D_{n}\right| \leqq \frac{1}{n^{2}} \sum_{i=1}^{k} \sum_{r=n_{i-1}+1}^{n_{i}}\left|s_{n_{i}}^{2}-s_{r}^{2}\right| .
$$

Using Schwartz's inequality we obtain for $n_{i-1}<r \leqq n_{i}$

$$
\begin{aligned}
& \text { m.e. }\left\{\left|s_{n_{i}}^{2}-s_{r}^{2}\right|\right\} \leqq\left(\text { m.e. }\left\{\left(s_{n_{i}}-s_{r}\right)^{2}\right\}\right)^{1 / 2}\left(\text { m.e. }\left\{\left(s_{n_{i}}+s_{r}\right)^{2}\right\}\right)^{1 / 2} \\
& =\left(n_{i}-r\right)^{1 / 2}\left(3 r+n_{i}\right)^{1 / 2}<2 n^{1 / 2}\left(n_{i}-r\right)^{1 / 2} \text {. }
\end{aligned}
$$

Thus

$$
\text { m.e. }\left\{\sum_{r=n_{i-1}+1}^{n_{i}}\left|s_{n_{i}}^{2}-s_{r}^{2}\right|\right\} \leqq 2 n^{1 / 2} \sum_{j=1}^{n_{i}-n_{i}-1} j^{1 / 2}<2 n^{1 / 2}\left(\frac{n}{k}+1\right)^{3 / 2}
$$

and therefore

7 Loc. cit. footnote 6, p. 561. This case corresponds to the problem of random walk in the presence of two absorbing barriers. 


$$
\text { m.e. }\left\{\left|D_{n}\right|\right\}<C / k^{1 / 2} \text {, }
$$

where $C$ is a certain constant.

This estimate of m.e. $\left\{\left|D_{n}\right|\right\}$ implies immediately that for $\epsilon>0$,

$$
\text { prob. }\left\{\left|D_{n}\right| \geqq \epsilon\right\} \leqq C / \epsilon k^{1 / 2} \text {. }
$$

We now write

$$
\text { prob. } \begin{aligned}
\left\{\frac{1}{n^{2}} \sum_{1}^{n} s_{r}^{2}<\alpha\right\}=\text { prob. } & \left\{\frac{1}{n^{2}} \sum_{1}^{n} s_{r}^{2}<\alpha,\left|D_{n}\right| \geqq \epsilon\right\} \\
& + \text { prob. }\left\{\frac{1}{n^{2}} \sum_{1}^{n} s_{\nu}^{2}<\alpha,\left|D_{n}\right|<\epsilon\right\}
\end{aligned}
$$

and notice that

prob. $\left\{\frac{1}{n^{2}} \sum_{1}^{n} s_{r}^{2}<\alpha,\left|D_{n}\right|<\epsilon\right\}$

$$
\leqq \text { prob. }\left\{\frac{1}{n^{2}} \sum_{1}^{k}\left(n_{i}-n_{i-1}\right) s_{n_{i}}^{2}<\alpha+\epsilon\right\} .
$$

Thus

$$
\text { prob. } \begin{aligned}
\left\{\frac{1}{n^{2}} \sum_{1}^{n} s_{r}^{2}\right. & <\alpha\} \\
& \leqq \frac{C}{\epsilon k^{1 / 2}}+\text { prob. }\left\{\frac{1}{n^{2}} \sum_{1}^{k}\left(n_{i}-n_{i-1}\right) s_{n_{i}}^{2}<\alpha+\epsilon\right\} .
\end{aligned}
$$

In exactly the same manner we obtain

$$
\text { prob. } \begin{aligned}
\left\{\frac{1}{n^{2}} \sum_{1}^{k}\left(n_{i}-n_{i-1}\right) s_{n_{i}}^{2}\right. & <\alpha-\epsilon\} \\
& \leqq \frac{C}{\epsilon k^{1 / 2}}+\text { prob. }\left\{\frac{1}{n^{2}} \sum_{1}^{n} s_{r}^{2}<\alpha\right\} .
\end{aligned}
$$

Combining (5) and (6) we can write

$$
\text { prob. } \begin{aligned}
\left\{\frac{1}{n^{2}} \sum_{1}^{k}\right. & \left.\left(n_{i}-n_{i-1}\right) s_{n_{i}}^{2}<\alpha-\epsilon\right\}-\frac{C}{\epsilon k^{1 / 2}} \\
& \leqq \text { prob. }\left\{\frac{1}{n^{2}} \sum_{1}^{n} s_{r}^{2}<\alpha\right\} \\
& \leqq \text { prob. }\left\{\frac{1}{n^{2}} \sum_{1}^{k}\left(n_{i}-n_{i-1}\right) s_{n_{i}}^{2}<\alpha+\epsilon\right\}+\frac{C}{\epsilon k^{1 / 2}}
\end{aligned}
$$


Let us now find the limit, as $n \rightarrow \infty$, of

$$
\text { prob. }\left\{\frac{1}{n^{2}} \sum_{1}^{k}\left(n_{i}-n_{i-1}\right) s_{n_{i}}^{2}<\beta\right\} \text {. }
$$

Denote by $\phi(\xi)$ the characteristic function of the distribution function of $X_{j}$.

The characteristic function of the joint distribution of

$$
\frac{n_{1}^{1 / 2}}{n} s_{n_{1}}, \frac{\left(n_{2}-n_{1}\right)^{1 / 2}}{n} s_{n_{2}}, \cdots, \frac{\left(n_{k}-n_{k-1}\right)^{1 / 2}}{n} s_{n_{k}},
$$

that is, the mathematical expectation of

$$
\exp \left\{\frac{i}{n} \sum_{1}^{k} \xi_{j}\left(n_{j}-n_{j-1}\right)^{1 / 2} s_{n_{j}}\right\}
$$

is easily found to be

$$
\prod_{j=1}^{k} \phi^{n_{j}-n_{j-1}}\left(\frac{1}{n} \sum_{i=j}^{k} \xi_{i}\left(n_{i}-n_{i-1}\right)^{1 / 2}\right) .
$$

If we let $n \rightarrow \infty$ (keeping $k$ fixed) we find easily that (10) approaches (uniformly in every bounded region of the $k$-dimensional space)

$$
\exp \left\{-\frac{1}{2 k^{2}} \sum_{j=1}^{k}\left(\sum_{i=j}^{k} \xi_{i}\right)^{2}\right\}
$$

which can be recognized as the characteristic function of the joint distribution of

$$
R_{1} / k, R_{2} / k, \cdots, R_{k} / k \text {. }
$$

(We recall that $R_{j}=G_{1}+\cdots+G_{j}$, where $G_{1}, G_{2}, \cdots, G_{k}$ are independent, normally distributed random variables each having mean 0 and standard deviation 1).

From the multidimensional continuity theorem for Fourier-Radon transforms, it follows that the joint distribution of the random variables (9) approaches the joint distribution of the random variables (11). Thus the probability (8) approaches

$$
\text { prob. }\left\{\frac{1}{k^{2}} \sum_{j=1}^{k} R_{j}^{2}<\beta\right\}=\rho_{k}(\beta) .
$$

If in (7) we let $n \rightarrow \infty$ we obtain the inequality 


$$
\begin{aligned}
\rho_{k}(\alpha-\epsilon)-\frac{C}{\epsilon k^{1 / 2}} & \leqq \liminf _{n \rightarrow \infty} \text { prob. }\left\{\frac{1}{n^{2}} \sum_{1}^{n} s_{r}^{2}<\alpha\right\} \\
& \leqq \limsup _{n \rightarrow \infty} \text { prob. }\left\{\frac{1}{n^{2}} \sum_{1}^{n} s_{r}^{2}<\alpha\right\} \\
& \leqq \rho_{k}(\alpha+\epsilon)+\frac{C}{\epsilon k^{1 / 2}},
\end{aligned}
$$

which is analogous to the inequality (1) of $\S 1$.

We complete the proof of III by showing that

$$
\rho_{k}(\beta) \rightarrow \sigma_{3}(\beta)
$$

for all $\beta$. It was shown by Cameron and Martin ${ }^{8}$ that the characteristic function of $\sigma_{3}(\beta)$ is

$$
\left(\sec (2 i \xi)^{1 / 2}\right)^{1 / 2}
$$

where $\sec (2 i \xi)^{1 / 2}$ is defined by the familiar infinite product

$$
\prod_{n=0}^{\infty}\left(1-\frac{2 i \xi}{((2 n+1) \pi / 2)^{2}}\right)^{-1}
$$

and $z^{1 / 2}$ is defined in the plane with negative real axis removed and satisfying the requirement that $z^{1 / 2}$ is positive for real and positive $z$.

It is then sufficient to show that the characteristic function

$$
\int_{0}^{\infty} e^{i \xi \beta} d \rho_{k}(\beta)
$$

approaches $\left(\sec (2 i \xi)^{1 / 2}\right)^{1 / 2}$ uniformly in every finite $\xi$-interval. We have

$$
\begin{aligned}
\int_{0}^{\infty} e^{i \xi \beta} d \rho_{k}(\beta)=(2 \pi)^{-k / 2} \int_{-\infty}^{\infty} \cdots \int_{-\infty}^{\infty} \exp \left\{\frac{i \xi}{k^{2}} \sum_{j=1}^{k}\left(\sum_{r=1}^{j} x_{r}\right)^{2}\right\} \\
\exp \left\{-\frac{1}{2} \sum_{1}^{k} x_{r}^{2}\right\} d x_{1} \cdots d x_{k} \\
=(2 \pi)^{-k / 2} \int_{-\infty}^{\infty} \cdots \int_{-\infty}^{\infty} \exp \left\{\frac{i \xi}{k^{2}} \sum_{1}^{k} y_{j}^{2}\right\} \\
\cdot \exp \left\{-\frac{y_{1}^{2}}{2}-\frac{1}{2} \sum_{2}^{k}\left(y_{j}-y_{j-1}\right)^{2}\right\} d y_{1} \cdots d y_{k} .
\end{aligned}
$$

${ }^{8}$ Loc. cit. footnote 4 . Actually, the characteristic function considered by Cameron and Martin is $\left(\sec (i \xi)^{1 / 2}\right)^{1 / 2}$ but their case corresponds to the normalization m.e. $\left\{G_{i}^{2}\right\}=1 / 2$ instead of m.e. $\left\{G_{i}^{2}\right\}=1$. 
If in the last integral we introduce the coordinate system corresponding to the "principal axes" of the quadratic form

$$
y_{1}^{2}+\sum_{2}^{k}\left(y_{j}-y_{j-1}\right)^{2}
$$

we notice readily that the integral will be reduced to the form

$$
(2 \pi)^{-k / 2} \int_{-\infty}^{\infty} \cdots \int_{-\infty}^{\infty} \exp \left\{\frac{i \xi}{k^{2}} \sum_{1}^{k} z_{j}^{2}\right\} \exp \left\{-\frac{1}{2} \sum_{1}^{k} \lambda_{j} z_{j}^{2}\right\} d z_{1} \cdots d z_{k},
$$

where $\lambda_{1}, \lambda_{2}, \cdots, \lambda_{k}$ are the eigenvalues of the matrix of the quadratic form (13). In the last integral the variables are separated and we obtain immediately

$$
\int_{0}^{\infty} e^{i \xi \beta} d \rho_{k}(\beta)=\prod_{j=1}^{k}\left(\lambda_{j}-\frac{2 i \xi}{k^{2}}\right)^{-1 / 2}
$$

The branch of

$$
\left(\lambda_{j}-2 i \xi / k^{2}\right)^{-1 / 2}
$$

is determined by removing from the complex $\xi$-plane the part of the imaginary axis between $-\infty$ and $-k^{2} \lambda_{j} / 2$ and requiring that (15) be positive for $\xi=y i, y>-k^{2} \lambda_{j} / 2$.

One could now calculate explicitly the eigenvalues $\lambda_{j}$ by elementary (but rather tedious) means and pass to the limit as $k \rightarrow \infty$. We prefer a less elementary method which has the advantage that it is applicable to many cases where the explicit calculation of eigenvalues is impossible.

We first notice that putting $\xi=0$ in (14) we obtain $\lambda_{1} \lambda_{2} \cdots \lambda_{k}=1$ and hence

$$
\int_{0}^{\infty} e^{i \xi \beta} d \rho_{k}(\beta)=\prod_{j=1}^{k}\left(1-\frac{2 i \xi}{k^{2} \lambda_{j}}\right)^{-1 / 2}
$$

Denoting by $\left(\left(a_{r s}\right)\right)$ the inverse matrix of the matrix of the quadratic form (13) we can verify directly that

$$
a_{r e}=\min (r, s) \text {. }
$$

Noticing that $1 / \lambda_{1}, \cdots, 1 / \lambda_{k}$ are the eigenvalues of $\left(\left(a_{r s}\right)\right)$ and using Hilbert's approach to Fredholm's theory ${ }^{9}$ we obtain that the quantities $1 / k^{2} \lambda_{j}$ approach the eigenvalues of the integral equation

- See D. Hilbert, Grundzilge einer allgemeinen Theorie der linearen Integralgleichungen, Berlin and Leipzig, Teubner, 1912. In particular see Theorem 2 on p. 14. 


$$
\int_{0}^{1} \min (s, t) f(t) d t=\lambda f(s)
$$

Writing (16) in the form

$$
\int_{0}^{0} t f(t) d t+s \int_{0}^{1} f(t) d t=\lambda f(s)
$$

and differentiating twice with respect to $s$ we obtain the differential equation

$$
\lambda f^{\prime \prime}(s)+f(s)=0
$$

subject to the boundary conditions $f(0)=f^{\prime}(1)=0$. Thus the eigenvalues are the numbers

$$
((2 n+1) \pi / 2)^{-2} \quad(n=0,1,2, \cdots)
$$

and it follows that

$$
\begin{aligned}
\lim _{k \rightarrow \infty} \int_{0}^{\infty} e^{i \xi \beta} d \rho_{k}(\beta) & =\prod_{n=0}^{\infty}\left(1-\frac{2 i \xi}{((2 n+1) \pi / 2)^{2}}\right)^{-1 / 2} \\
& =\left(\sec (2 i \xi)^{1 / 2}\right)^{1 / 2}
\end{aligned}
$$

where the determination of $\left(\sec (2 i \xi)^{1 / 2}\right)^{1 / 2}$ was described above. ${ }^{10}$ This completes the proof of III.

The proof of IV proceeds as follows. Denoting by $Q_{n}(\alpha)$ the probability

$$
\text { prob. }\left\{n^{-8 / 2} \sum_{1}^{n}\left|s_{r}\right|<\alpha\right\}
$$

we are led to the inequality

$$
\begin{aligned}
\text { prob. } & \left\{k^{-3 / 2} \sum_{1}^{k}\left|R_{j}\right|<\alpha-\epsilon\right\}-\frac{C_{1}}{\epsilon k^{1 / 2}} \leqq \liminf _{n \rightarrow \infty} Q_{n}(\alpha) \\
\leqq & \limsup _{n \rightarrow \infty} Q_{n}(\alpha) \leqq \text { prob. }\left\{k^{-3 / 2} \sum_{1}^{k}\left|R_{j}\right|<\alpha+\epsilon\right\}+\frac{C_{1}}{\epsilon k^{1 / 2}}
\end{aligned}
$$

We then chose particular random variables whose distribution is given by the formula

$$
\text { prob. }\left\{X_{j}<\alpha\right\}=\frac{1}{2^{1 / 2}} \int_{-\infty}^{\alpha} \exp \left(-2^{1 / 2}|u|\right) d u \text {. }
$$

${ }^{10}$ That convergence in (17) is uniform in every finite $\xi$-interval also follows from Hilbert's considerations quoted above. 
For these random variables it was proved (loc. cit. footnote 2 ) that

$$
\lim _{n \rightarrow \infty} Q_{n}(\alpha)=\sigma_{4}(\alpha) .{ }^{11}
$$

We complete the proof for the general case in the same way as in the proof of $I$.

UNIVERSITY OF MICHIGAN AND

CORNELL UNIVERSITY

11 Inasmuch as we have not proved that $\sigma_{4}$ is continuous this statement should be qualified by adding that it holds at each continuity point of $\sigma_{4}$.

Added in proof: In the meantime Dr. Erdös succeeded in proving that $\sigma_{4}$ is everywhere continuous. The proof is quite involved and will not be reproduced here. 